\title{
Article \\ Postharvest UV-B and Photoperiod with Blue + Red LEDs as Strategies to Stimulate Carotenogenesis in Bell Peppers
}

\author{
Lorena Martínez-Zamora, Noelia Castillejo (D) and Francisco Artés-Hernández * (D) \\ Postharvest and Refrigeration Group, Department of Agronomical Engineering \& Institute of \\ Plant Biotechnology, Universidad Politécnica de Cartagena, Cartagena, 30203 Murcia, Spain; \\ lorena.martinez@upct.es (L.M.-Z.); noelia.castillejo@upct.es (N.C.) \\ * Correspondence: fr.artes-hdez@upct.es; Tel.: +34-968-325509
}

Citation: Martínez-Zamora, L.; Castillejo, N.; Artés-Hernández, F. Postharvest UV-B and Photoperiod with Blue + Red LEDs as Strategies to Stimulate Carotenogenesis in Bell Peppers. Appl. Sci. 2021, 11, 3736 https://doi.org/10.3390/app11093736

Academic Editor: Wojciech

Kolanowski

Received: 29 March 2021

Accepted: 19 April 2021

Published: 21 April 2021

Publisher's Note: MDPI stays neutral with regard to jurisdictional claims in published maps and institutional affiliations.

Copyright: (c) 2021 by the authors. Licensee MDPI, Basel, Switzerland. This article is an open access article distributed under the terms and conditions of the Creative Commons Attribution (CC BY) license (https:/ / creativecommons.org/licenses/by/ $4.0 /)$.
Featured Application: Illumination with blue + red LEDs during the nights of retail sale periods is recommended to increase carotenogenesis in bell peppers. This night-time photoperiod combined with a low postharvest UV-B dose in a logistic centre may present a synergistic effect on the carotenoid accumulation in these fruits.

Abstract: Background: Our objective was to evaluate carotenoid accumulation in bell peppers during shelf life under different light conditions. Methods: Fruit stored for $6 \mathrm{~d}$ at $7^{\circ} \mathrm{C}$ received a $9 \mathrm{~kJ} \mathrm{~m}^{-2}$ UV-B treatment, while non-UV-treated were used as control (CTRL). Subsequently, all peppers were disposed for a retail sale period of $4 \mathrm{~d}$ at $20{ }^{\circ} \mathrm{C}$ with a photoperiod of $14 \mathrm{~h}$ under fluorescent light (FL) $+10 \mathrm{~h}$ under darkness (D), FL, or blue + red LEDs (BR LED). Results: Total antioxidant capacity (TAC) was increased by the UV-B treatment and the photoperiods supplemented with FL and BR LED, which was directly related to the carotenoid content. In fact, CTRL peppers ( $225 \mathrm{mg} \beta$-carotene $\mathrm{kg}^{-1}$ ) under FL+BR LED showed an increase of $\sim 33 \%$ of 13 -cis- $\beta$-carotene, $\sim 24 \%$ of all-trans- $\beta$-carotene, and $\sim 27.5 \%$ of 9 -cis- $\beta$-carotene compared to FL $+\mathrm{D}$ and FL + FL. Capsaicinoids showed an increase by $\sim 22 \%, \sim 38 \%$, and $\sim 27 \%$ in the content of capsanthin, capsanthin laurate, and capsanthin esters, respectively, after the UV-B treatment, which was even enhanced after the LED-supplemented photoperiod by $\sim 18 \%$ compared to FL+D. Conclusions: Illumination with BR LEDs + UV-B during the retail sale period nights is recommended to increase the bioactive content of bell peppers via carotenoid accumulation to $270 \mathrm{mg} \beta$-carotene $\mathrm{kg}^{-1}$.

Keywords: Capsicum annum L.; ultraviolet; lighting emitting diode; abiotic stress; bioactive compounds; nutraceuticals; antioxidants; shelf life

\section{Introduction}

Fruit and vegetables are essential in human health due to the high content in bioactive compounds [1]. Indeed, these nutraceuticals have been associated with a reduction in the risk of chronic and pro-inflammatory diseases [2]. Red peppers (Capsicum annuum L.) in particular are demonstrated to be an important source of carotenoids [3], flavonoids, and vitamins [4], being a powerful ingredient able to fight against ageing and prevent chronic diseases [5]. In this context, Spain is the fifth largest producer of bell peppers in the world after China, Mexico, Turkey, and Indonesia [6] and the first European exporter country [7] with 1.4 million tons.

As light is an essential factor directly related to the synthesis of primary and secondary metabolites [8], the bioaccumulation pathways of these compounds can vary with changes in the quantity and quality of lighting applied. In fact, application of abiotic stresses throughout non-visible spectral regions, such as UV-B $(280-315 \mathrm{~nm})$, are reported to have an hormetic effect characterized by positive stimulation of the metabolite pathways under low doses $[9,10]$, but a negative response of the plant tissues may appear under moderate to high UV doses [11]. In fact, UV RESPONSE LOCUS 8 (UVR8), as the main UV-B receptors, 
are responsible for regulating carotenoid biosynthesis [12]. Thus, UV-B has shown to improve the contents of carotenoids and flavonoids as the main antioxidant compounds in bell peppers [13]. In this way, UV can be used to induce the expression of antioxidant enzymes, which increase the biosynthesis of vitamins, flavonoids, phenolic acids, and carotenoids, among others [14-16].

From another point of view, the environmental conditions in the final steps of the fresh bell pepper retail chain in supermarkets widely apply conventional fluorescent lamps $\left(2 \mathrm{~W} \mathrm{~m}^{-2}\right.$ on the floor and $8 \mathrm{~W} \mathrm{~m}^{-2}$ on the shelves) for $14 \mathrm{~h}$ between 15 to $22{ }^{\circ} \mathrm{C}$ [17]. However, when supermarkets close, the lamps above the horticultural commodities are usually switched off for approximately the next $10 \mathrm{~h}$ in order to save energy, with such conditions biologically promoting the accumulation of phytochemicals. In recent years, the use of coloured LEDs in supermarkets and the industry have increased due to their low energy requirements and their possibility to customize the light intensity and spectral properties. In this sense, the use of postharvest LED lighting has already shown potential beneficial effects in broccoli florets [18-20], brussels sprouts [21], and broccoli sprouts [22].

However, the effect in quality and phytochemicals accumulation of horticultural commodities of the combination of different postharvest lighting conditions from the visible and non-visible spectrum is mostly unexplored. Pérez-Ambrocio et al. [23] combined postharvest blue LED and UV-C lighting obtaining promising results in habanero pepper (Capsicum chinense) by increasing chlorophyll, carotenoid, capsaicin, phenolic, and flavonoid content.

The combination of UV illumination and a photoperiod with lights from the visible spectrum during the postharvest shelf life of fruit and vegetables could improve the bioactive compound contents without compromising other quality attributes, or even improving them. Therefore, the aim of the present work was to evaluate the effect on carotenoid accumulation of UV-B radiation treatment jointly with a photoperiod under different illumination spectra during the commercialization of red bell peppers.

\section{Materials and Methods}

\subsection{Plant Material}

The selected bell pepper (Capsicum annum L.) variety was 'Angus' from Syngenta España. S.A. (Torre-Pacheco, Murcia, Spain) of the California type and with red ripening. Bell peppers were grown in a greenhouse in the Southeast of Spain by a local producer under integrated pest management (Tárraga y Henarejos, S.L., Murcia, Spain) and harvested on 21st July 2020, when the maturity index reached $21.8 \pm 0.7$. Quality characterization at harvest of the studied fruit is shown in Table 1.

Table 1. Main quality parameters at harvest of studied bell peppers $(n=25)$.

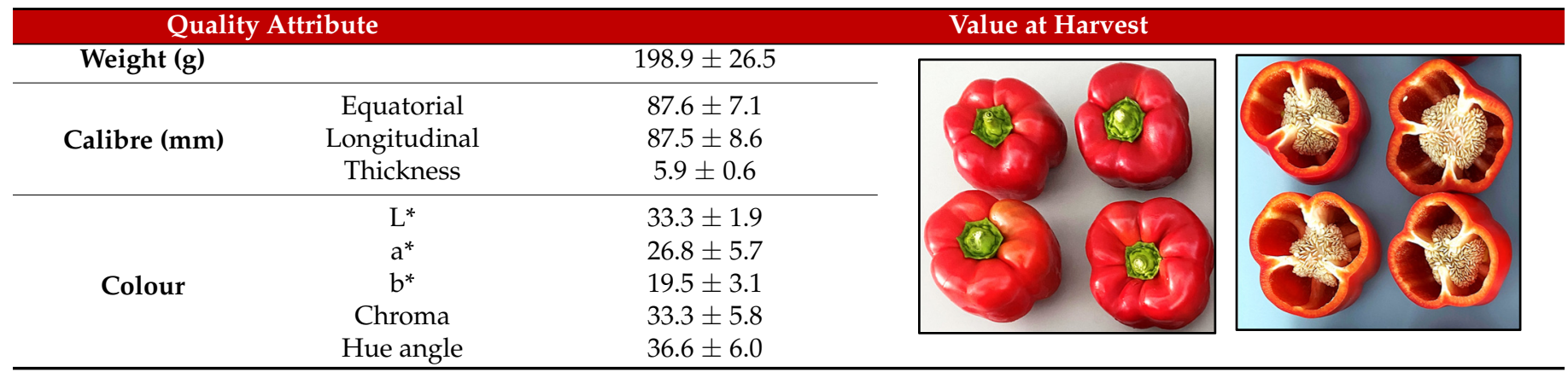

Plant material was transported for $30 \mathrm{~min}$ to the Universidad Politécnica of Cartagena. After reception, the healthy and undamaged peppers were selected and washed with 5\% peracetic acid (Citrocide ${ }^{\circledR} \mathrm{PC}$, Citrosol, Valencia, Spain) at $7^{\circ} \mathrm{C}$ for $2 \mathrm{~min}$ and rinsed in tap water for $1 \mathrm{~min}$. A total of 550 bell peppers were washed and dried with absorbent paper. 


\subsection{Postharvest Treatments and Light Photoperiod}

Bell red peppers were stored for $6 \mathrm{~d}$ at $7{ }^{\circ} \mathrm{C}$ and $80 \%$ relative humidity (RH) under conditions of darkness, simulating a short postharvest storage + commercial exportation within Europe. Then, considering that the fruit arrived at a logistic platform, or even though that they could be repacked, peppers were divided into two groups: CTRL and UV-B. UV-B red peppers were exposed to $9 \mathrm{~kJ} \mathrm{~m}^{-2} \mathrm{UV}-\mathrm{B}(19 \mathrm{~min} 30 \mathrm{~s}$ ) in a radiation chamber consisted of a reflective stainless-steel chamber described by Formica-Oliveira et al. [24], which was equipped with 6 UV-B unfiltered emitting lamps (TL 40W/01 RS; Philips, Eindhoven, The Netherlands). The applied UV-B intensity was calculated as the mean of 20 readings with a LP 471 UVB radiometer (Delta OHM, Padua, Italy): $8.94 \pm 0.40 \mathrm{~W} \mathrm{~m}^{-2}$, and this dose was chosen based on our preliminary test and findings by Formica-Oliveira et al. [10,24].

After treatment, bell peppers were kept at $20{ }^{\circ} \mathrm{C}$ and $60 \% \mathrm{RH}$ during 2, 3, and $4 \mathrm{~d}$, simulating a retail sale period in supermarkets on the destination country. In order to adapt our model to what usually happens in supermarkets, three different photoperiods were applied following the same pattern: $14 \mathrm{~h}$ under fluorescent lighting (FL) with $403 \mathrm{~kJ} \mathrm{~m}^{-2}$ when supermarkets are open $+10 \mathrm{~h}$ under the next variable lighting treatment when supermarkets are close at night)

- D: darkness, as it is usually performed.

- FL: fluorescent lighting with lower energy $\left(280 \mathrm{~kJ} \mathrm{~m}^{-2}\right)$ to save costs provided by fluorescent lamps (OSRAM DULUX L 36W/840, Munich, Germany) with broad white spectrum and $8 \mathrm{~W} \mathrm{~m}^{-2}$, simulating the conventional storage conditions measured by us for this study in several Spanish supermarkets during June-July 2020.

- BR LED: a combination of blue + red LEDs (LEDMurcia S.L., Murcia, Spain) with $576 \mathrm{~kJ} \mathrm{~m}^{-2}$. LED lamps were applied with a simultaneous combination (1:1) of blue (peak at $450 \mathrm{~nm}$ ) and red (peak at $660 \mathrm{~nm}$ ). This combination was chosen due to our previous preliminary test based on previous findings by Martínez-Zamora et al. [25] and Pennisi et al. [26].

A constant photosynthetic photon flux density (PPFD) for FL and LED lights of 36.7 and $73.1 \mu \mathrm{mol} \mathrm{m} \mathrm{m}^{-2} \mathrm{~s}^{-1}$, respectively, were measured using a Quantum-Photo Radiometer Data Logger DO 9721 (Delta Ohm, S.R.L., Venice, Italy). Quality sampling days throughout the studied postharvest period were at harvest (day 0 ), after $6 \mathrm{~d}$ at $7^{\circ} \mathrm{C}$ (end of the simulated storage/transportation period), and after 2,3 , and $4 \mathrm{~d}$ at $20^{\circ} \mathrm{C}$ of the simulated retail sale period. A schematic representation of this study is shown in Figure 1.

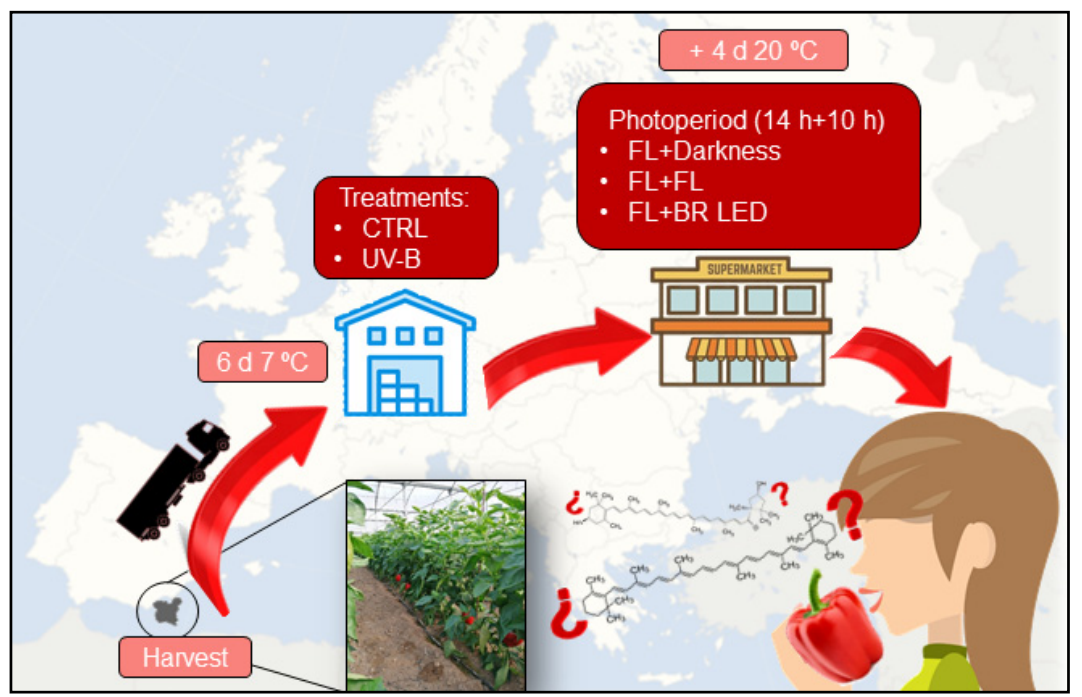

Figure 1. Schematic representation of the experimental design of the study. 


\subsection{Physicochemical and Sensory Quality Determination}

The physicochemical quality analyses were carried out on each sampling day. Bell peppers were weighed (g) using a FH-2000 scale (GRAM, Barcelona, Spain). Weight losses $(\%)$ were calculated by using the next formula: ((Initial Weight-Final Weight)/Initial Weight $) \times 100$. Thickness and longitudinal and equatorial calibre were initially measured using a digital calliper (Mitutoyo, Neuss, Germany), expressing the results in mm. Colour was determined using a Konica Minolta CR-400 colorimeter (Tokyo, Kanto, Japan). Measurements were recorded using the CIELab system $\left(\mathrm{L}^{*}, \mathrm{a}^{*}\right.$, and $\mathrm{b}^{*}$ coordinates). Total colour differences $(\Delta \mathrm{E})$ throughout the shelf life were compared to their respective initial values according to equations previously described by Torres-Sánchez et al. [27]. A texturometer (Brookfield, CT3-4500, Toronto, ON, Canada) was used to test firmness at room temperature (RT) with a cylinder of $8 \mathrm{~mm}$ diameter and a surface area of $2.01 \mathrm{~cm}^{2}$ and compressing the fruit by $5 \mathrm{~mm}$ at a contact speed of $2 \mathrm{~mm} \mathrm{~s}^{-1}$, expressing these results in newtons $(\mathrm{N})$.

After that, bell peppers were cut and blended using a Robot Coupe J80 Ultra (Vincennes, Île-de-France, France). The total soluble solids content (TSS), pH, and titratable acidity (TA) of the obtained pepper juice were determined. TSS were measured by a digital handheld refractometer (Atago N1; Tokyo, Kanto, Japan) and expressed as \%. A pH meter (GLP21, Crison; Alella, Cataluña, Spain) was used to determine the $\mathrm{pH}$ while TA was determined by titration of $5 \mathrm{~mL}$ of pepper juice plus $45 \mathrm{~mL}$ of distilled water with $0.1 \mathrm{M}$ $\mathrm{NaOH}$ to $\mathrm{pH} 8.1$ (T50, Metter Toledo; Milan, Lombardia, Italy) and expressed as mg citric acid $100 \mathrm{~mL}^{-1}$.

Each sample was analysed in quintuplicate on each sampling day per treatment, and 5 bell peppers formed a replicate $(n=25)$. After the physicochemical analyses were performed, three replicates of 5 juiced peppers each per treatment were frozen in liquid nitrogen for every sampling time and stored at $-80^{\circ} \mathrm{C}$. Frozen samples were ground to fine powder prior to analyses using liquid $\mathrm{N}_{2}$ with a mincer (IKA, A 11 basic, Berlin, Germany) at $12,700 \mathrm{~g}$ for $10 \mathrm{~s}$.

The characterization was completed with a sensory evaluation each sampling day. The tasting room was air-conditioned and free of disturbing factors. Bell peppers were cut into $1 \times 5 \mathrm{~mm}$ pieces. Ten panellists were trained following the ISO guide [28]. Samples were coded with three random digits and were presented individually to the panellists. Mineral water was provided for mouth rinsing between samples. The attributes measured for the colour, odour, and taste characteristics were 'Dehydration', 'Visual appearance', 'Colour (green/mature)', 'Odour', 'Firmness (touch, when slightly compressing with the fingers)'. 'Firmness (mouth, when eating)', and 'Flavour'. Overall acceptability was measured by a panel of 30 consumers. A hedonic scale in intensity from 1 to 5 was used, being 1 'Extremely bad', 2 'Bad'; 3 'Limit of commercialization', 4 'Good', and 5 'Excellent'.

\subsection{Extraction and Determination of Bioactive Compounds}

One gram of bell pepper powder was placed in plastic tubes and $10 \mathrm{~mL}$ methanol: $\mathrm{H}_{2} \mathrm{O}$ $(80: 20, v / v)$ was added. The extraction was carried out in an orbital shaker (Stuart, Stone, UK), where the samples were vigorously shaken for $1 \mathrm{~h}$ at $4{ }^{\circ} \mathrm{C}$ in darkness. The extracts were centrifuged at $3220 \times \mathrm{g}$ for $10 \mathrm{~min}$ at $5{ }^{\circ} \mathrm{C}$ and the supernatant was collected and kept at $-80{ }^{\circ} \mathrm{C}$ until further analysis of total phenolic content (TPC), total flavonoid content (TFC), and total antioxidant capacity (TAC). Extractions were carried out on each sampling day in triplicate.

The TPC was determined as previously described by Castillejo et al. [22]. For that, $19 \mu \mathrm{L}$ sample extract were mixed with $29 \mu \mathrm{L}$ of $1 \mathrm{~mol} \mathrm{~L}^{-1}$ Folin-Ciocalteu reagent. Three minutes later, $192 \mu \mathrm{L}$ of $0.4 \% \mathrm{Na}_{2} \mathrm{CO}_{3}$ and $2 \% \mathrm{NaOH}$ were added. After $1 \mathrm{~h}$ incubation in darkness, the absorbance was measured at $750 \mathrm{~nm}$ using a microplate reader (Tecan Infinite M200, Männedorf, Switzerland). The TPC was expressed as g gallic acid equivalents (GAE) $\mathrm{kg}^{-1}$ fresh weight $(\mathrm{fw})$. Each sample was analysed in triplicate.

The TFC was determined following the method described by Hamed et al. [5]. Briefly, $0.030 \mathrm{~mL}$ of extract were mixed with $80 \mu \mathrm{L}$ of aluminium chloride $\left(20 \mathrm{~g} \mathrm{~L}^{-1}\right)$. The samples 
were shaken for $30 \mathrm{~s}$ and then incubated in darkness for $1 \mathrm{~h}$. After that, the reaction absorbance was measured at $415 \mathrm{~nm}$. TFC was expressed as g rutin equivalents (RE) $\mathrm{kg}^{-1}$ of fw. Each sample was analysed in triplicate on each sampling day.

The TAC was analysed following the DPPH procedure [29]. DPPH assay was performed by adding $194 \mu \mathrm{L}$ of DPPH $(0.7 \mathrm{mM})$ solution to $21 \mu \mathrm{L}$ of bell pepper extract. After $30 \mathrm{~min}$ incubation in darkness, absorbance was measured at $515 \mathrm{~nm}$. Obtained data were expressed as $\mathrm{g}$ of trolox equivalents (TE) $\mathrm{kg}^{-1} \mathrm{fw}$. Each sample was analysed in triplicate on each sampling day.

\subsection{Extraction and Determination of Carotenoids}

Preparation and carotenoid analysis were performed according to Martínez-Zamora et al. [25] with slight modifications. A sample of $1 \mathrm{~g}$ was homogenized with $5 \mathrm{~mL}$ of chloroform:dichloromethane $(2: 1, v / v)$ in a basic grinder (IKA A11, Staufen, Germany). The extraction was carried out in an orbital shaker (Stuart, Stone, UK) for $20 \mathrm{~min}$ at $200 \mathrm{rpm}$ at $4{ }^{\circ} \mathrm{C}$. After this time, $2 \mathrm{~mL}$ of $1 \mathrm{M} \mathrm{NaCl}$ solution were added for phase separation. The extracts were centrifuged at $5000 \times \mathrm{g}$ for $10 \mathrm{~min}$ at $4{ }^{\circ} \mathrm{C}$ and the organic phase was collected. The supernatant was re-extracted by adding $2.5 \mathrm{~mL}$ of chloroform:dichloromethane $(2: 1$, $v / v$ ) and centrifuging again, and the organic phase was collected. Finally, the extracted phase was dried by centrifugal evaporation and re-dissolved in $4 \mathrm{~mL}$ methanol:MTBE $(25: 75, v / v)$ and filtered using $0.2 \mu \mathrm{m}$ PTFE membrane filters.

An ultrahigh-performance liquid chromatography (UHPLC) instrument (Shimadzu, Kyoto, Japan) equipped with a DGU-20A degasser, LC-30AD quaternary pump, SIL-30AC autosampler, CTO-10AS column heater, and SPDM-20A photodiode array detector was used. Chromatographic analyses were carried out into a C30 column $(250 \mathrm{~mm} \times 4.6 \mathrm{~mm}$, $3 \mu \mathrm{m}$ particle size; YMC Co., Kyoto, Japan). Carotenoids were quantified as equivalents of $\beta$-carotene. The results were expressed as $\mathrm{mg} \mathrm{kg}^{-1} \mathrm{fw}$. Each sample was analysed in triplicate.

\subsection{Data Analysis}

The experiment was a three-factor (treatment $-\mathrm{T}-\times$ photoperiod $-\mathrm{P}-\times$ time $-\mathrm{t}-$ ) design subjected to analysis of variance (ANOVA) using Statgraphics Plus software (v. 5.1. Statpoint Technologies. Inc., Warrenton, VA, USA). Statistical significance was assessed at the level $p<0.05$, and Tukey's multiple range test was used to separate means.

\section{Results}

\subsection{Physicochemical Quality Changes throughout Shelf Life}

Bell pepper weight at harvest was $198.9 \pm 26.5 \mathrm{~g}$ (Table 1 ), for which $0.5 \%$ weight losses were reported after $6 \mathrm{~d}$ at $7^{\circ} \mathrm{C}$ (Table 2). Although there were no weight loss differences after $6 \mathrm{~d}$ at $7{ }^{\circ} \mathrm{C}$ after the UV-B treatment for CTRL fruit, dehydration was higher after the retail sale period of $4 \mathrm{~d}$ at $20^{\circ} \mathrm{C}$, especially under the FL + BR LED photoperiod. Hence, such losses are directly related to firmness, which was slightly lower under the FL + FL and $\mathrm{FL}+\mathrm{BR}$ LED photoperiods and accentuated after $4 \mathrm{~d}$ at $20^{\circ} \mathrm{C}$ by $\sim 40 \%-50 \%$ reductions (Table 2).

The optimum ripening stage as a non-climacteric fruit was reached after $6 \mathrm{~d}$ at $7{ }^{\circ} \mathrm{C}$, which can be observed when the $\Delta \mathrm{E}$ achieves constant values just before the retail sale period at $20^{\circ} \mathrm{C}$ (Table 2). In this way, a slight colour variation in fruit under the FL + FL photoperiod was shown regarding peppers stored under FL+D and FL+BR LED. TSS also showed a slightly increase throughout the shelf life, especially on UV-B-treated samples. Indeed, the interaction among all variables $(\mathrm{T}, \mathrm{P}$, and $\mathrm{t}$ ) of the experimental design was highly significant, which also occurs with TA and MI (TSS/TA). While TA decreased throughout shelf life, MI increased, especially in CTRL red peppers under the FL+FL photoperiod, reaching values of $0.28 \pm 0.05 \mathrm{mg}$ citric acid $100 \mathrm{~mL}^{-1}$ and $28.7 \pm 0.6$ for MI (Table 2). In contrast, $\mathrm{pH}$ values remained quite constant during shelf life at $4.9 \pm 0.0$ and no interaction was found among dependent variables. 
Table 2. Physicochemical quality attributes of UV-B-treated and untreated CTRL bell peppers after $6 \mathrm{~d}$ at $7^{\circ} \mathrm{C}$ followed by a retail sale period of 2,3 , and $4 \mathrm{~d}$ at $20^{\circ} \mathrm{C}$ under a photoperiod of $14 \mathrm{~h}$ in fluorescent light $+10 \mathrm{~h}$ in different lighting conditions.

\begin{tabular}{|c|c|c|c|c|c|c|c|c|c|}
\hline & $\mathrm{T}$ & $\begin{array}{c}P \\
14 h+10 h\end{array}$ & $\mathbf{t}$ & $\underset{(1)}{\text { Weight Losses }}$ & $\underset{(2)}{\text { Firmness }}$ & $\Delta \mathrm{E}$ & $\underset{(3)}{\text { TSS }}$ & $\underset{(4)}{\mathrm{TA}}$ & TSS/TA \\
\hline At har & & & $0 \mathrm{~d}$ & - & $19.8 \pm 7.3$ & - & $7.5 \pm 0.4$ & $0.35 \pm 0.02$ & $21.8 \pm 0.7$ \\
\hline After $6 \mathrm{~d}$ at & CTRL & - & & $0.5 \pm 0.1$ & $19.7 \pm 4.5$ & $8.4 \pm 6.1$ & $7.6 \pm 0.3$ & $0.35 \pm 0.03$ & $21.7 \pm 1.1$ \\
\hline $7^{\circ} \mathrm{C}$ & UV-B & - & $6 \mathrm{~d}$ & $0.5 \pm 0.1$ & $17.2 \pm 3.6$ & $5.9 \pm 3.9$ & $7.6 \pm 0.3$ & $0.31 \pm 0.01$ & $24.7 \pm 1.6$ \\
\hline \multirow{25}{*}{$\begin{array}{c}\text { After a } \\
\text { retail sale } \\
\text { period at } \\
20^{\circ} \mathrm{C}\end{array}$} & \multirow{9}{*}{ CTRL } & \multirow{3}{*}{$\mathrm{FL}+\mathrm{D}$} & $+2 \mathrm{~d}$ & $1.1 \pm 0.2$ & $17.2 \pm 2.6$ & $6.2 \pm 2.6$ & $7.8 \pm 0.2$ & $0.30 \pm 0.02$ & $26.2 \pm 1.1$ \\
\hline & & & $+3 d$ & $2.5 \pm 0.5$ & $13.8 \pm 3.3$ & $6.4 \pm 2.6$ & $7.5 \pm 0.1$ & $0.28 \pm 0.02$ & $26.5 \pm 1.2$ \\
\hline & & & $+4 \mathrm{~d}$ & $3.7 \pm 0.7$ & $10.1 \pm 2.0$ & $6.3 \pm 2.2$ & $8.1 \pm 0.3$ & $0.32 \pm 0.01$ & $25.6 \pm 0.2$ \\
\hline & & & $+2 \mathrm{~d}$ & $1.3 \pm 0.3$ & $16.7 \pm 3.0$ & $7.4 \pm 5.8$ & $7.9 \pm 0.2$ & $0.36 \pm 0.02$ & $21.8 \pm 0.7$ \\
\hline & & \multirow[t]{2}{*}{$\mathrm{FL}+\mathrm{FL}$} & $+3 \mathrm{~d}$ & $2.8 \pm 0.6$ & $13.7 \pm 3.0$ & $7.7 \pm 4.6$ & $7.6 \pm 0.3$ & $0.31 \pm 0.01$ & $24.4 \pm 0.6$ \\
\hline & & & $+4 \mathrm{~d}$ & $4.0 \pm 0.8$ & $9.9 \pm 2.2$ & $7.4 \pm 5.7$ & $7.8 \pm 0.3$ & $0.28 \pm 0.05$ & $28.7 \pm 0.6$ \\
\hline & & \multirow{3}{*}{ FL + BR LED } & $+2 d$ & $1.5 \pm 0.2$ & $15.2 \pm 3.2$ & $6.3 \pm 3.5$ & $7.7 \pm 0.2$ & $0.30 \pm 0.01$ & $25.3 \pm 1.2$ \\
\hline & & & $+3 \mathrm{~d}$ & $3.1 \pm 0.5$ & $13.7 \pm 3.4$ & $6.0 \pm 2.4$ & $7.6 \pm 0.2$ & $0.31 \pm 0.02$ & $24.3 \pm 1.4$ \\
\hline & & & $+4 \mathrm{~d}$ & $4.6 \pm 0.7$ & $8.1 \pm 2.1$ & $6.2 \pm 2.6$ & $7.7 \pm 0.1$ & $0.31 \pm 0.01$ & $25.1 \pm 1.1$ \\
\hline & \multirow{9}{*}{ UV-B } & \multirow{3}{*}{$F L+D$} & $+2 \mathrm{~d}$ & $1.2 \pm 0.3$ & $16.7 \pm 4.0$ & $6.1 \pm 2.7$ & $7.7 \pm 0.2$ & $0.32 \pm 0.01$ & $23.7 \pm 0.8$ \\
\hline & & & $+3 \mathrm{~d}$ & $2.5 \pm 0.6$ & $14.4 \pm 3.2$ & $6.6 \pm 2.8$ & $7.5 \pm 0.1$ & $0.30 \pm 0.01$ & $25.2 \pm 1.2$ \\
\hline & & & $+4 \mathrm{~d}$ & $3.7 \pm 0.9$ & $9.7 \pm 2.6$ & $6.2 \pm 2.3$ & $7.8 \pm 0.2$ & $0.30 \pm 0.01$ & $26.4 \pm 0.1$ \\
\hline & & \multirow{4}{*}{$\mathrm{FL}+\mathrm{FL}$} & $+2 \mathrm{~d}$ & $1.3 \pm 0.2$ & $17.4 \pm 3.3$ & $8.5 \pm 5.6$ & $7.6 \pm 0.5$ & $0.31 \pm 0.02$ & $24.7 \pm 0.7$ \\
\hline & & & $+3 d$ & $2.7 \pm 0.4$ & $12.2 \pm 2.9$ & $8.1 \pm 5.4$ & $7.6 \pm 0.1$ & $0.29 \pm 0.02$ & $26.4 \pm 2.1$ \\
\hline & & & $+4 \mathrm{~d}$ & $4.0 \pm 0.5$ & $9.1 \pm 1.8$ & $6.8 \pm 4.7$ & $7.7 \pm 0.3$ & $0.30 \pm 0.01$ & $25.7 \pm 1.0$ \\
\hline & & & $+2 \mathrm{~d}$ & $1.5 \pm 0.2$ & $14.5 \pm 2.5$ & $7.1 \pm 3.4$ & $7.5 \pm 0.3$ & $0.27 \pm 0.03$ & $27.8 \pm 1.7$ \\
\hline & & \multirow[t]{2}{*}{ FL + BR LED } & $+3 d$ & $3.1 \pm 0.4$ & $11.2 \pm 2.5$ & $6.8 \pm 4.1$ & $7.8 \pm 0.2$ & $0.33 \pm 0.06$ & $24.1 \pm 0.5$ \\
\hline & & & $+4 \mathrm{~d}$ & $4.7 \pm 0.6$ & $8.4 \pm 1.8$ & $6.5 \pm 2.5$ & $8.1 \pm 0.2$ & $0.31 \pm 0.00$ & $25.7 \pm 0.9$ \\
\hline & & $\mathrm{T}$ & & n.s. & n.s. & n.s. & $(0.042)^{*}$ & $(0.004)^{* *}$ & n.s. \\
\hline & & $\mathrm{P}$ & & $(0.139) * * *$ & $(0.758) * * *$ & $(1.039)$ * & n.s. & n.s. & n.s. \\
\hline & & $\mathrm{t}$ & & $(0.139)^{* * *}$ & $(0.758)^{* * *}$ & n.s. & $(0.052)^{* * *}$ & $(0.005)^{* * *}$ & $(0.477)^{* * *}$ \\
\hline & & $\mathrm{T} \times \mathrm{P}$ & & n.s. & n.s. & n.s. & $(0.074)^{* * *}$ & $(0.008)^{* * *}$ & $(0.675)^{* * * *}$ \\
\hline & & $\mathrm{T} \times \mathrm{t}$ & & n.s. & n.s. & n.s. & $(0.074)^{* * *}$ & $(0.008) * * *$ & $(0.675) * *$ \\
\hline & & $P \times t$ & & $(0.241) * *$ & n.s. & n.s. & $(0.090)^{* * *}$ & $(0.009)^{* * *}$ & $(0.827)^{* * * *}$ \\
\hline & & $\mathrm{T} \times \mathrm{P} \times \mathrm{t}$ & & n.s. & n.s. & n.s. & $(0.127)^{* * *}$ & $(0.013)^{* * *}$ & $(1.169)^{* * *}$ \\
\hline
\end{tabular}

FL: fluorescent light; D: darkness; BR LED: blue and red LEDs. Data are vertically compared. T: treatment; P: photoperiod; t: time (days). ${ }^{*} p<0.05 ;{ }^{* *} p<0.005 ;{ }^{* * *} p<0.001$; n.s.: no significant differences. ${ }^{(1)}(\%) ;{ }^{(2)} \mathrm{N} ;{ }^{(3)}(\%) ;{ }^{(4)} \mathrm{mg}$ citric acid $100 \mathrm{~mL}^{-1}$.

These physicochemical quality parameters can be related to sensory perception of red peppers (Figure 2), which did not report differences among the studied treatments and photoperiods. In this way, 'Colour' increased by 2 points according to the evaluation scale after the retailing period (Table 2). 'Firmness' (both when slightly compressing with fingers and when eating) experimented an important decrease after $6 \mathrm{~d} 7^{\circ} \mathrm{C}+4 \mathrm{~d} 20^{\circ} \mathrm{C}$ which was directly related to dehydration, justified with the objective quality attributes of weight losses and firmness shown in Table 2. As observed, these parameters also affected the acceptability of the fruit at the end of the retail sale period, being close to the limit of commercialization, giving then some extra days to consumers. In our previous unpublished shelf life experiments with this 'Angus' cv, a shelf life of $15 \mathrm{~d}$ was mainly reached being the first 6-8 d under refrigeration conditions simulating the storage + transportation period.

\subsection{Bioactive Compound Content and Total Antioxidant Capacity}

Total phenolic content of red peppers at harvest was $2.12 \pm 0.11 \mathrm{~g} \mathrm{GAE} \mathrm{kg}^{-1}$, which decreased by $\sim 20 \%$ after $6 \mathrm{~d}$ at $7{ }^{\circ} \mathrm{C}$, although such an initial amount was again reconstituted after the retailing period at $20^{\circ} \mathrm{C}$, especially under the FL + BR LED photoperiod (Table 3). In contrast, flavonoid biosynthesis was not affected by the photoperiod nor UV-B treatment, increasing its content at harvest by $\sim 18 \%$ in CTRL fruit after $6 \mathrm{~d}$ at $7{ }^{\circ} \mathrm{C}+4 \mathrm{~d}$ at $20^{\circ} \mathrm{C}$. As total phenolic and flavonoid contents have been directly related to the antioxidant capacity of these fruit, the TAC was positively affected by FL + FL and FL + BR LED photoperiods regarding $\mathrm{FL}+\mathrm{D}$, which was also highly remarkable after $6 \mathrm{~d}$ at $7{ }^{\circ} \mathrm{C}+4 \mathrm{~d} 20^{\circ} \mathrm{C}(p<0.05)$. 


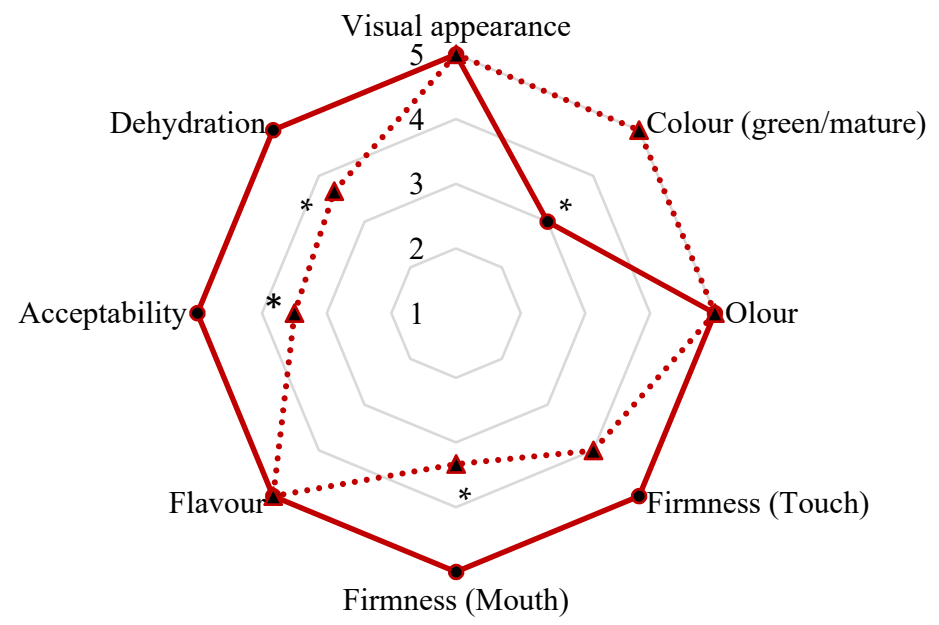

$\longrightarrow$ At harvest $\quad \cdots * 6 \mathrm{~d} 77^{\circ} \mathrm{C}+4 \mathrm{~d} 20^{\circ} \mathrm{C}$

Figure 2. Sensory analysis of untreated and UV-B-treated bell peppers after $6 \mathrm{~d}$ at $7^{\circ} \mathrm{C}$ and after a complimentary retail sale period of $4 \mathrm{~d}$ at $20^{\circ} \mathrm{C}$ as mean values under all light conditions, which did not reported differences. ${ }^{*}$ denotes statistical differences $(p<0.05)$. A 5-point hedonic scale was used, with 1 meaning 'Extremely bad', 3 'Limit of commercialization', and 5 'Excellent'.

Table 3. Total phenolic compounds ( $\mathrm{g}$ gallic acid equivalents $\mathrm{kg}^{-1}$ ), total flavonoid compounds ( $\mathrm{g}$ rutin equivalents $\mathrm{kg}^{-1}$ ), and total antioxidant capacity ( $\mathrm{g}$ trolox equivalents $\mathrm{kg}^{-1}$ ) of UV-B-treated and untreated (CTRL) bell peppers during a storage and transportation period of $6 \mathrm{~d}$ at $7{ }^{\circ} \mathrm{C}$ followed by a retail sale period of 2,3 , or $4 \mathrm{~d}$ at $20^{\circ} \mathrm{C}$ under a photoperiod of $14 \mathrm{~h}$ in fluorescent light $+10 \mathrm{~h}$ in different light conditions.

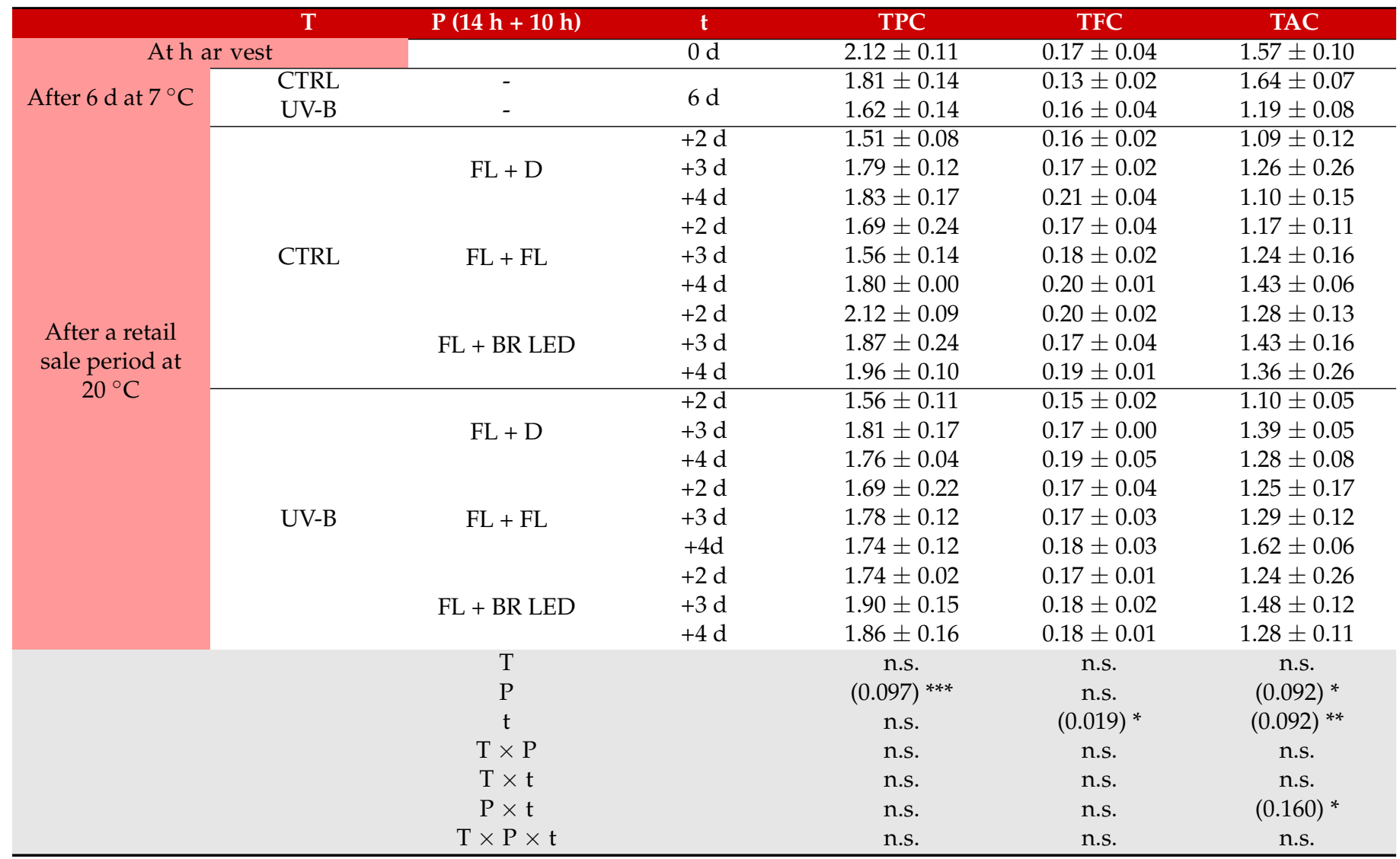

TPC: total phenolic compounds; TFC: total flavonoid compounds; TAC: total antioxidant capacity; FL: fluorescent light; D: darkness; BR LED: blue and red LEDs. Data are vertically compared. T: treatment; P: photoperiod; t: time (days). ${ }^{*} p<0.05 ;{ }^{* *} p<0.005 ;{ }^{* * *} p<0.001 ;$ n.s.: no significant differences. 


\subsection{Carotenoid Biosynthesis}

As it was presumed with TAC values, the carotenoid biosynthesis was highly enhanced by FL + FL and FL+BR LED photoperiods, especially after a low UV-B dose (Table 4). $\beta$-Carotene and its derivatives were those reporting a higher bio stimulation with UV-B, mainly when it was combined with the lighting conditions of a supermarket (Figure 3). Capsaicinoids were the main carotenoid compounds found in red peppers. Their content was also positively affected with the synergistic effect of a postharvest UV-B treatment and a FL+BR LED photoperiod during the retail sale period (Figure 4). In this sense, a general increase of the total carotenoid content after UV-B postharvest treatment and photoperiods is shown in Figure 5.

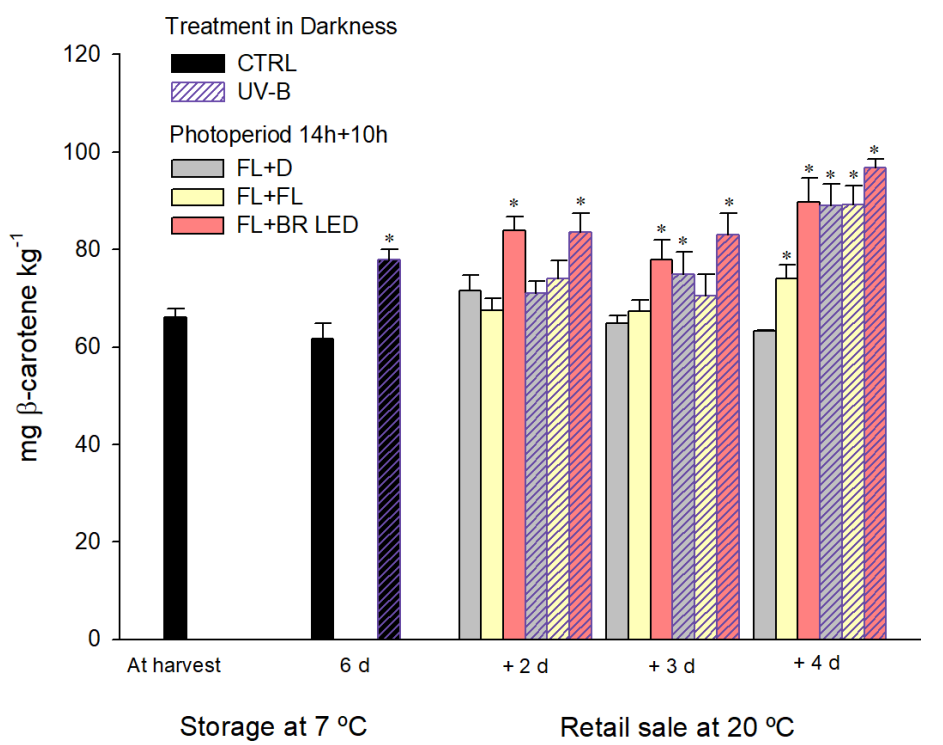

Figure 3. Total $\beta$-carotene content of UV-B-treated and untreated CTRL bell peppers after $6 \mathrm{~d}$ at $7{ }^{\circ} \mathrm{C}$ followed by a retail sale period of 2,3 , and $4 \mathrm{~d}$ at $20{ }^{\circ} \mathrm{C}$ under different lighting conditions. *: significant differences compared to CTRL. Treatment in darkness (T): CTRL (non-striped bars) and UV-B (striped bars); Photoperiod during retail sale (P): FL+D (grey bars), FL+FL (yellow bars),

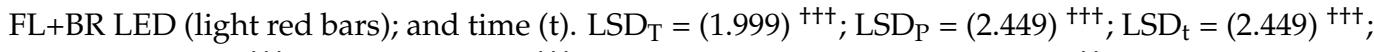
$\mathrm{LSD}_{\mathrm{T} \times \mathrm{P}}=(3.463)^{+++} ; \mathrm{LSD}_{\mathrm{T} \times \mathrm{t}}=(3.463)^{++\dagger} ; \mathrm{LSD}_{\mathrm{P} \times \mathrm{t}}=$ n.s.; $\mathrm{LSD}_{\mathrm{T} \times \mathrm{P} \times \mathrm{t}}=(5.998)^{+\dagger}$. n.s.: no significant; ${ }^{++}: p>0.005{ }^{+++}: p>0.001$. 


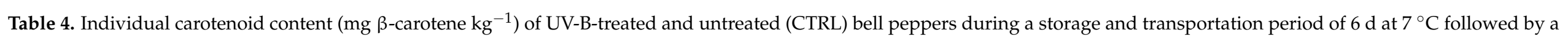
retail sale period of 2,3 , or $4 \mathrm{~d}$ at $20^{\circ} \mathrm{C}$ under a photoperiod of $14 \mathrm{~h}$ in fluorescent light $+10 \mathrm{~h}$ in different light conditions.

\begin{tabular}{|c|c|c|c|c|c|c|c|c|c|c|c|c|}
\hline & $\mathrm{T}$ & $P(14 h+10 h)$ & $\mathbf{t}$ & $\begin{array}{l}\text { All-Trans- } \\
\text { Neoxanthin }\end{array}$ & $\begin{array}{l}\text { All-Trans- } \\
\text { Lutein }\end{array}$ & Capsanthin & $\begin{array}{c}\text { Capsanthin } \\
\text { Laurate }\end{array}$ & $\begin{array}{l}\text { 13-Cis- } \beta- \\
\text { Carotene }\end{array}$ & $\begin{array}{l}\text { All-Trans- } \\
\beta \text {-Carotene }\end{array}$ & $\begin{array}{l}\text { 9-Cis- } \beta \text { - } \\
\text { Carotene }\end{array}$ & $\begin{array}{c}\text { Capsanthin } \\
\text { Myristate }\end{array}$ & $\begin{array}{l}\text { Capsanthin } \\
\text { Esters }\end{array}$ \\
\hline \multicolumn{2}{|c|}{ At harvest } & & $0 \mathrm{~d}$ & $9.9 \pm 0.7$ & $2.3 \pm 0.3$ & $54.6 \pm 4.8$ & $7.8 \pm 0.3$ & $19.0 \pm 0.9$ & $35.2 \pm 0.6$ & $6.2 \pm 0.2$ & $7.7 \pm 0.1$ & $77.5 \pm 2.7$ \\
\hline \multirow{2}{*}{$\begin{array}{c}\text { After } 6 \mathrm{~d} \text { at } \\
\quad 7^{\circ} \mathrm{C} \\
\end{array}$} & CTRL & - & \multirow[b]{2}{*}{$6 \mathrm{~d}$} & $9.6 \pm 1.8$ & $2.2 \pm 0.3$ & $39.5 \pm 0.8$ & $8.2 \pm 0.0$ & $15.3 \pm 0.5$ & $33.5 \pm 1.6$ & $6.8 \pm 0.9$ & $10.5 \pm 1.1$ & $80.2 \pm 6.9$ \\
\hline & UV-B & - & & $10.8 \pm 0.2$ & $2.2 \pm 0.3$ & $48.0 \pm 4.5^{\mathrm{A}}$ & $11.3 \pm 0.4^{\mathrm{A}}$ & $19.6 \pm 0.8^{\mathrm{A}}$ & $36.6 \pm 1.6$ & $7.2 \pm 0.6$ & $10.4 \pm 1.1$ & $102.2 \pm 7.2^{\mathrm{A}}$ \\
\hline \multirow{23}{*}{$\begin{array}{l}\text { After a } \\
\text { retail sale } \\
\text { period at } \\
20^{\circ} \mathrm{C}\end{array}$} & \multirow{10}{*}{ CTRL } & \multirow{4}{*}{$\mathrm{FL}+\mathrm{D}$} & $+2 \mathrm{~d}$ & $10.5 \pm 0.2$ & $2.4 \pm 0.1$ & $54.7 \pm 0.7$ & $9.7 \pm 0.7$ & $18.4 \pm 1.3$ & $33.9 \pm 3.1$ & $7.8 \pm 0.1$ & $8.5 \pm 0.8$ & $82.7 \pm 3.3$ \\
\hline & & & $+3 \mathrm{~d}$ & $14.0 \pm 0.2$ & $2.8 \pm 0.0$ & $51.9 \pm 0.0$ & $10.0 \pm 0.3$ & $16.9 \pm 0.7$ & $30.9 \pm 0.7$ & $6.6 \pm 0.4$ & $8.1 \pm 0.5$ & $76.5 \pm 2.9$ \\
\hline & & & $+4 \mathrm{~d}$ & $15.3 \pm 1.2$ & $3.1 \pm 0.2$ & $78.1 \pm 3.4$ & $9.9 \pm 0.8$ & $17.2 \pm 0.7$ & $28.8 \pm 0.4$ & $8.4 \pm 0.2$ & $8.4 \pm 0.1$ & $75.9 \pm 7.7$ \\
\hline & & & $+2 \mathrm{~d}$ & $10.1 \pm 0.3$ & $2.5 \pm 0.2$ & $58.5 \pm 5.8$ & $8.7 \pm 0.4$ & $17.9 \pm 0.4$ & $31.6 \pm 1.7$ & $7.5 \pm 0.1$ & $7.4 \pm 0.5$ & $80.0 \pm 2.4$ \\
\hline & & \multirow[t]{2}{*}{$\mathrm{FL}+\mathrm{FL}$} & $+3 \mathrm{~d}$ & $14.2 \pm 1.0$ & $2.7 \pm 0.1$ & $52.0 \pm 2.3$ & $10.4 \pm 0.5$ & $19.6 \pm 1.0$ & $31.3 \pm 1.4$ & $6.2 \pm 0.1$ & $8.0 \pm 0.2$ & $94.9 \pm 2.6$ \\
\hline & & & $+4 \mathrm{~d}$ & $15.8 \pm 1.0$ & $2.9 \pm 0.1$ & $84.6 \pm 2.4$ & $10.5 \pm 0.7$ & $20.0 \pm 1.0$ & $34.2 \pm 1.9$ & $8.3 \pm 0.4$ & $9.4 \pm 0.3$ & $81.7 \pm 2.3$ \\
\hline & & \multirow{3}{*}{$\mathrm{FL}+\mathrm{BR}$ LED } & $+2 d$ & $14.1 \pm 1.3$ & $2.8 \pm 0.2$ & $70.3 \pm 4.5$ & $13.2 \pm 0.0$ & $24.5 \pm 1.8$ & $43.8 \pm 2.9$ & $9.8 \pm 0.4$ & $10.5 \pm 0.0$ & $108.1 \pm 7.2$ \\
\hline & & & $+3 \mathrm{~d}$ & $14.4 \pm 1.8$ & $2.7 \pm 0.2$ & $70.5 \pm 5.6$ & $9.8 \pm 0.4$ & $24.0 \pm 2.2$ & $32.3 \pm 1.7$ & $9.4 \pm 0.6$ & $9.3 \pm 0.7$ & $90.6 \pm 8.8$ \\
\hline & & & $+4 \mathrm{~d}$ & $18.2 \pm 0.7$ & $3.5 \pm 0.2$ & $102.4 \pm 4.3$ & $12.7 \pm 1.1$ & $24.6 \pm 1.3$ & $42.2 \pm 2.7$ & $9.4 \pm 0.5$ & $9.8 \pm 0.4$ & $88.6 \pm 2.5$ \\
\hline & & \multirow{4}{*}{$\mathrm{FL}+\mathrm{D}$} & $+2 \mathrm{~d}$ & $9.6 \pm 0.4$ & $2.2 \pm 0.0$ & $69.2 \pm 0.6$ & $9.9 \pm 0.9$ & $18.3 \pm 0.5$ & $34.3 \pm 2.8$ & $7.1 \pm 0.5$ & $8.6 \pm 0.5$ & $83.8 \pm 5.6$ \\
\hline & \multirow{6}{*}{ UV-B } & & $+3 d$ & $14.2 \pm 0.2$ & $2.8 \pm 0.1$ & $67.1 \pm 1.0$ & $10.7 \pm 0.4$ & $19.3 \pm 0.9$ & $35.7 \pm 2.1$ & $7.7 \pm 1.0$ & $10.4 \pm 0.7$ & $81.6 \pm 12.3$ \\
\hline & & & $+4 \mathrm{~d}$ & $17.7 \pm 0.5$ & $3.4 \pm 0.0$ & $78.7 \pm 2.1$ & $12.1 \pm 0.5$ & $23.7 \pm 1.4$ & $41.5 \pm 2.4$ & $9.8 \pm 0.8$ & $11.0 \pm 0.9$ & $90.7 \pm 8.2$ \\
\hline & & & $+2 \mathrm{~d}$ & $9.9 \pm 0.3$ & $2.7 \pm 0.1$ & $67.8 \pm 2.1$ & $10.3 \pm 0.2$ & $18.5 \pm 0.4$ & $36.6 \pm 1.9$ & $7.2 \pm 0.5$ & $10.8 \pm 0.5$ & $91.1 \pm 6.6$ \\
\hline & & $\mathrm{FL}+\mathrm{FL}$ & $+3 \mathrm{~d}$ & $13.9 \pm 0.9$ & $2.8 \pm 0.2$ & $68.5 \pm 3.6$ & $9.8 \pm 1.1$ & $18.0 \pm 0.2$ & $33.7 \pm 3.2$ & $7.6 \pm 0.3$ & $9.3 \pm 0.8$ & $77.2 \pm 6.3$ \\
\hline & & \multirow[t]{2}{*}{$\mathrm{FL}+\mathrm{BR}$ LED } & $+3 d$ & $16.5 \pm 0.7$ & $3.1 \pm 0.2$ & $81.1 \pm 3.9$ & $12.7 \pm 1.3$ & $21.3 \pm 0.3$ & $38.8 \pm 2.5$ & $8.3 \pm 0.7$ & $11.1 \pm 0.7$ & $92.8 \pm 9.1$ \\
\hline & & & $+4 \mathrm{~d}$ & $18.1 \pm 0.2$ & $3.5 \pm 0.0$ & $98.3 \pm 4.3$ & $13.3 \pm 0.5$ & $26.1 \pm 0.1$ & $45.9 \pm 1.3$ & $10.0 \pm 0.4$ & $11.5 \pm 1.2$ & $97.1 \pm 6.3$ \\
\hline & & $\mathrm{T}$ & & $(0.461) *$ & $(0.079)^{* * *}$ & $(2.017)^{* * *}$ & $(0.401)^{* * *}$ & $(0.602) *$ & $(1.204)^{* * *}$ & n.s. & $(0.343)^{* * *}$ & n.s. \\
\hline & & $\mathrm{P}$ & & $(0.564)^{* * *}$ & $(0.097)^{* * *}$ & $(2.470)^{* * *}$ & $(0.491)^{* * *}$ & $(0.737)^{* * *}$ & $(1.474)^{* * *}$ & $(0.360)^{* * * *}$ & $(0.420)^{* * *}$ & $(4.370)^{* * *}$ \\
\hline & & $\mathrm{t}$ & & $(0.564)^{* * *}$ & $(0.097)^{* * *}$ & $(2.470)^{* * *}$ & $(0.491)^{* * *}$ & $(0.737)^{* * *}$ & $(1.474)^{* * *}$ & $(0.360)^{* * *}$ & $(0.420)^{*}$ & n.s. \\
\hline & & $\mathrm{T} \times \mathrm{P}$ & & n.s. & n.s. & $(3.493)^{*}$ & n.s. & $(1.042)^{* * *}$ & $(2.085)^{* *}$ & $(0.509) * *$ & n.s. & n.s. \\
\hline & & $\mathrm{T} \times \mathrm{t}$ & & n.s. & n.s. & $(3.493)^{* * *}$ & $(0.694) *$ & $(1.042)^{* * *}$ & $(2.085)^{* * *}$ & $(0.509)^{* * *}$ & n.s. & $(6.179) * *$ \\
\hline & & $\mathrm{P} \times \mathrm{t}$ & & $(0.978)^{* * *}$ & $(0.167) *$ & $(4.278) * *$ & $(0.851) *$ & n.s. & $(2.553) *$ & n.s. & n.s. & n.s. \\
\hline & & $\mathrm{T} \times \mathrm{P} \times \mathrm{t}$ & & $(1.382) *$ & $(0.237)$ * & n.s. & $(1.203)^{* * *}$ & n.s. & $(3.611)^{* *}$ & $(0.881)$ * & $(1.030)^{* * *}$ & $(10.703)^{* * *}$ \\
\hline
\end{tabular}

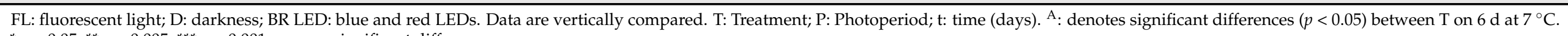
${ }^{*} p<0.05 ; * *<0.005 ;{ }^{* * *} p<0.001 ;$ n.s.: no significant differences. 


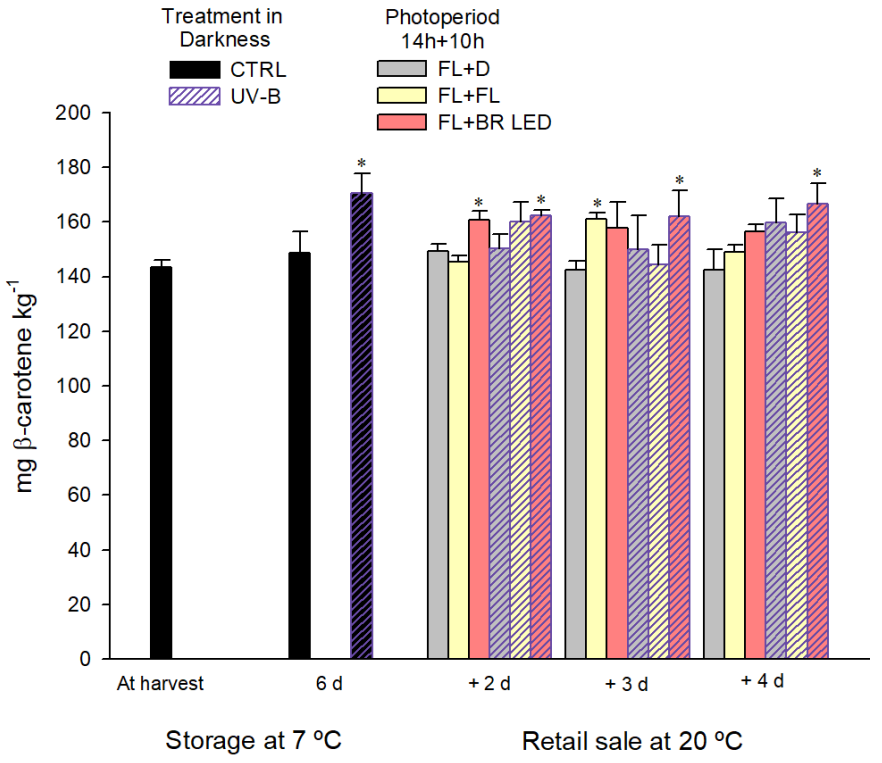

Figure 4. Total capsanthin content of UV-B-treated and untreated CTRL bell peppers after $6 \mathrm{~d}$ at $7{ }^{\circ} \mathrm{C}$ followed by a retail sale period of 2,3 , and $4 \mathrm{~d}$ at $20^{\circ} \mathrm{C}$ under different lighting conditions. *: significant differences compared to CTRL. Treatment in darkness (T): CTRL (non-striped bars) and UV-B (striped bars); Photoperiod during retail sale (P): FL + D (grey bars), FL + FL (yellow bars), $\mathrm{FL}+\mathrm{BR}$ LED (light red bars); and time (t). $\mathrm{LSD}_{\mathrm{T}}=$ n.s.; $\mathrm{LSD}_{\mathrm{P}}=(4.577){ }^{++} ; \mathrm{LSD}_{\mathrm{t}}=$ n.s.; $\mathrm{LSD}_{\mathrm{T} \times \mathrm{P}}=$ n.s.; $\mathrm{LSD}_{\mathrm{T} \times \mathrm{t}}=(6.473)^{\dagger}{ }^{\dagger} \mathrm{LSD}_{\mathrm{P} \times \mathrm{t}}=$ n.s.; $\mathrm{LSD}_{\mathrm{T} \times \mathrm{P} \times \mathrm{t}}=(11.211)^{++t}$. n.s.: no significant; ${ }^{+}: p>0.05$; ${ }^{++}: p>0.005 ;{ }^{+++}: p>0.001$.

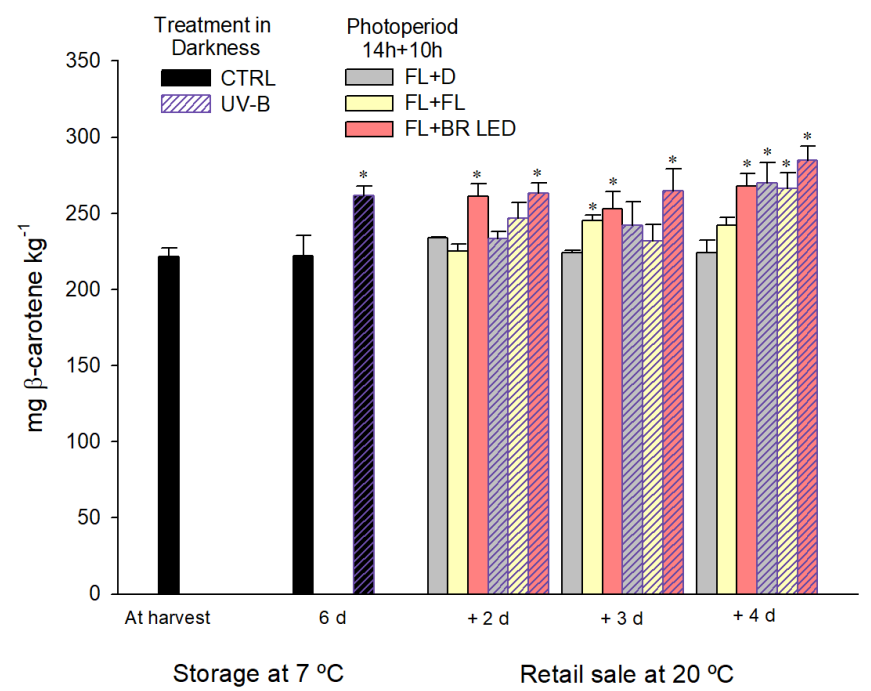

Figure 5. Total carotenoid content of untreated and UV-B-treated red peppers during $6 \mathrm{~d}$ at $7{ }^{\circ} \mathrm{C}$ followed by commercialization of 2,3 , and $4 \mathrm{~d}$ at $20{ }^{\circ} \mathrm{C}$ under different light conditions measured by UHPLC. *: significant differences compared to CTRL. Treatment in darkness (T): CTRL (non-striped bars) and UV-B (striped bars); Photoperiod during retail sale (P): FL+D (grey bars), FL+FL (yellow bars), FL+BR LED (light red bars); and time (t). $\mathrm{LSD}_{\mathrm{T}}=(5.255){ }^{+++} ; \mathrm{LSD}_{\mathrm{P}}=(6.435){ }^{+++} ; \mathrm{LSD}_{\mathrm{t}}=(6.435)$ ${ }^{++t} ; \mathrm{LSD}_{\mathrm{T} \times \mathrm{P}}=(9.101)^{t++} ; \mathrm{LSD}_{\mathrm{T} \times \mathrm{t}}=(9.101)^{t++} ; \mathrm{LSD}_{\mathrm{P} \times \mathrm{t}}=$ n.s.; $\mathrm{LSD}_{\mathrm{T} \times \mathrm{P} \times \mathrm{t}}=(15.764)^{t+}$. n.s.: no significant; ${ }^{+}: p>0.05{ }^{{ }^{++}}: p>0.005{ }^{+++}: p>0.001$.

At harvest, red peppers reported a total of $221.7 \pm 5.5 \mathrm{mg} \beta$-carotene $\mathrm{kg}^{-1}$, which was maintained during the refrigerated period (Figure 5). Capsaicinoids represented the $64.5 \%$ of the total carotenoid content, followed by $\beta$-carotene and its derivatives (30\%), all-trans-neoxanthin (4.5\%), and all-trans-lutein (1\%) (Table 4). 
All-trans-neoxanthin and all-trans-lutein compounds showed a similar behaviour, being positively affected by UV-B treatment, the photoperiod with light combinations, and the retailing period at $20{ }^{\circ} \mathrm{C}$. In fact, UV-B-treated and untreated red peppers after the supplementary $4 \mathrm{~d}$ at $20^{\circ} \mathrm{C}$ under FL+BR LED photoperiod registered the same content of these compounds, but UV-B red peppers exhibited an increase of $\sim 15 \%$ and $\sim 10 \%$ under $\mathrm{FL}+\mathrm{D}$ and $\mathrm{FL}+\mathrm{FL}$, respectively.

All-trans- $\beta$-carotene and 13-cis- $\beta$-carotene biosynthesis was slightly increased after $9 \mathrm{~kJ} \mathrm{~m}^{-2}$ UV-B treatment, which was especially remarkable on the same day of the treatment. In contrast, no differences were found in the accumulation of 9 -cis- $\beta$-carotene after the UV-B treatment. Nevertheless, all $\beta$-carotene derivatives were positively affected by the FL + BR LED photoperiod, which was highly observed after the supplementary $4 \mathrm{~d}$ at $20^{\circ} \mathrm{C}$. In fact, as a mean of the retailing period, CTRL red peppers under FL+BR LED showed an increase of $\sim 33 \%$ of 13 -cis- $\beta$-carotene, $\sim 24 \%$ of all-trans- $\beta$-carotene, and $\sim 27.5 \%$ of 9-cis- $\beta$-carotene compared to FL $+\mathrm{D}$ and FL + FL. To a lesser extent, UV-B red peppers under FL + BR LED photoperiod also reported $\sim 11.5 \%, \sim 8.5 \%$, and $\sim 11 \%$ more accumulation of these compounds, respectively, than FL + D and FL + FL. Moreover, no differences were found between CTRL and UV-B red peppers under the same photoperiod, alternating $14 \mathrm{~h}$ FL and $10 \mathrm{~h}$ BR LED lighting.

All-trans-neoxanthin and all-trans-lutein compounds showed a similar behaviour, being positively affected by UV-B treatment, the photoperiod with light combinations, and the retailing period at $20{ }^{\circ} \mathrm{C}$. In fact, UV-B-treated and untreated red peppers after the supplementary $4 \mathrm{~d}$ at $20^{\circ} \mathrm{C}$ under FL + BR LED photoperiod registered the same content of these compounds, but UV-B red peppers exhibited an increase of $\sim 15 \%$ and $~ 10 \%$ under $\mathrm{FL}+\mathrm{D}$ and FL $+\mathrm{FL}$, respectively.

All-trans- $\beta$-carotene and 13-cis- $\beta$-carotene biosynthesis was slightly increased after $9 \mathrm{~kJ} \mathrm{~m}{ }^{-2}$ UV-B treatment, which was especially remarkable on the same day of the treatment. In contrast, no differences were found in the accumulation of 9 -cis- $\beta$-carotene after the UV-B treatment. Nevertheless, all $\beta$-carotene derivatives were positively affected by the FL + BR LED photoperiod, which was highly observed after the supplementary $4 \mathrm{~d}$ at $20{ }^{\circ} \mathrm{C}$. In fact, as a mean of the retailing period, CTRL red peppers under FL+BR LED showed an increase of $\sim 33 \%$ of 13 -cis- $\beta$-carotene, $\sim 24 \%$ of all-trans- $\beta$-carotene, and $\sim 27.5 \%$ of 9-cis- $\beta$-carotene compared to FL $+\mathrm{D}$ and FL + FL. To a lesser extent, UV-B red peppers under FL + BR LED photoperiod also reported $\sim 11.5 \%, \sim 8.5 \%$, and $\sim 11 \%$ more accumulation of these compounds, respectively, than FL + D and FL + FL. Moreover, no differences were found between CTRL and UV-B red peppers under the same photoperiod, alternating $14 \mathrm{~h}$ FL and $10 \mathrm{~h}$ BR LED lighting.

Concerning the major carotenoid compounds, capsanthin and its derivatives were also bio stimulated by the UV-B treatment, photoperiod, and the time at $20^{\circ} \mathrm{C}$. Indeed, immediately after the $9 \mathrm{~kJ} \mathrm{~m}-2 \mathrm{UV}$-B dose applied on $6 \mathrm{~d}$ at $7^{\circ} \mathrm{C}$, red peppers showed an amelioration effect in the accumulation of capsanthin, capsanthin laurate, and capsanthin esters by $\sim 22 \%, \sim 38 \%$, and $\sim 27 \%$, respectively. After that, as a mean of the capsanthin content during the retailing period at $20{ }^{\circ} \mathrm{C}, \mathrm{CTRL}$ red peppers under FL + BR LED showed an increase by $\sim 31.5 \%$ and $\sim 25 \%$ regarding FL $+\mathrm{D}$ and FL + FL, respectively. Accordingly, capsanthin laurate and capsanthin myristate were enhanced by $\sim 20.5 \%$ and $\sim 19.3 \%$, respectively, compared to FL+D and FL+FL. Moreover, the amount of capsanthin esters was enhanced after the FL+BR LED photoperiod in $\sim 22 \%$ compared to FL+D and in $\sim 12 \%$ compared to FL+FL photoperiod.

UV-B-treated red peppers under the supplemented $10 \mathrm{~h}$ BR LED photoperiod showed an increase of $\sim 18 \%$ and $\sim 13 \%$ in the capsanthin content regarding FL $+\mathrm{D}$ and FL $+\mathrm{FL}$, while capsanthin laurate, capsanthin myristate, and capsanthin esters were increased by $\sim 14.7 \%, \sim 9.5 \%$, and $\sim 8.1 \%$, respectively, compared to the other tested photoperiods.

From a general point of view, a $10 \mathrm{~h}$ photoperiod supplemented with BR LED was demonstrated to be as effective as a carotenoid biosynthesis stimulator, compared to turning off the lights at night during the retail sale period, and without compromising the quality. 
An additional postharvest light stress of $9 \mathrm{~kJ} \mathrm{~m}^{-2} \mathrm{UV}-\mathrm{B}$ even increased such accumulation. In fact, a combination of both UV-B treatment and a photoperiod under FL+BR LED has shown a symbiotic effect during the retailing period concerning the accumulation of carotenes, lutein, and neoxanthin, due to their amount being similar under single and combined light conditions. Nevertheless, a synergistic behaviour was observed regarding the capsanthin accumulation and its esters ( $60 \%$ of the total carotenoid content). This fact can be due to the stimulation of the carotenoid biosynthesis, as phytochemicals involved in the plant photoprotection, throughout several biochemical pathways.

\section{Discussion}

The studied bell peppers showed $\sim 27 \%$ more water losses than those under the FL+D photoperiod. This fact can be related to previous results obtained by Pennisi et al. [30], who recently showed similar losses in fresh-cut red chard (26.8\%-38.2\%) and rocket leaves $\left(22 \%\right.$ and $31 \%$ ) after $10 \mathrm{~d}$ at $5{ }^{\circ} \mathrm{C}$ in response to monochromatic red and blue LEDs $\left(35 \mu \mathrm{mol} \mathrm{m}{ }^{-2} \mathrm{~s}^{-1}\right)$, respectively. Such low weight losses are in agreement to previous results reported by Kasim and Kasim [31], who reported $1 \%$ dehydration after $6 \mathrm{~d}$ at $7{ }^{\circ} \mathrm{C}$, whose data were not affected after UV-B treatments of 4.46 and $8.93 \mathrm{~kJ} \mathrm{~m}^{-2}$. Similar results were obtained by Miranda-Molina et al. [4], who showed weight losses higher than $50 \%$ in serrano chilli peppers after $33 \mathrm{~d}$ at $25^{\circ} \mathrm{C}$.

Regarding colour variations $(\Delta \mathrm{E})$, this effect is similar to results reported by Kasim and Kasim, [31], who showed that low UV-B doses $\left(4.46 \mathrm{~kJ} \mathrm{~m}^{-2}\right)$ did not affect the colour development during $6 \mathrm{~d}$ at $7{ }^{\circ} \mathrm{C}$. As observed, TSS has been related to MI and, particularly in red peppers, these two parameters have also been associated with colour development [32], which can also be contrasted with our present results. Moreover, sensory quality values can be related to the overall marketability, which has been previously reported to be increased after applying blue and red LEDs [33] or UV low doses [31,34].

As total phenolic and flavonoid contents have been directly related to the antioxidant capacity of these fruit [5], the TAC was positively affected by FL + FL and FL + BR LED photoperiods regarding $\mathrm{FL}+\mathrm{D}$, which was also highly remarkable after $6 \mathrm{~d}$ at $7{ }^{\circ} \mathrm{C}+4 \mathrm{~d}$ $20^{\circ} \mathrm{C}$. Values obtained can be comparable to previous studies in which UV-B increased the TAC according to DPPH values [31], and this effect has also been shown in UV-C-treated red peppers [35]. Moreover, an increase of the TAC measured by FRAP in fresh-cut red peppers for $11 \mathrm{~d}$ at $7{ }^{\circ} \mathrm{C}$ has also been reported for the application of $8 \mathrm{~h}$ blue and red LEDs separately [33]. Nevertheless, there are no previous studies combining both UV-B and LED photoperiod to study their synergistic effect. Indeed, this behaviour can be explained by the biosynthesis of nutraceuticals as carotenoids, which were potentiated by both postharvest strategies as it is subsequently detailed.

The carotenoid profile of the studied red peppers is in agreement with previous studies in different varieties of red peppers reported by Hassan et al. [3].

Our hypothesis to explain the basis of this positive effect on the carotenogenesis of carotenes and xanthophylls in red bell peppers, which especially occurs during fruit ripening, is that it is controlled by transcript genes which are mainly regulated by light incidence and high temperatures [36]. As the initial precursor of lycopene in the top of the carotenoid chain, phytoene synthase (PSY) plays an essential role in the conversion of geranylgeranyl diphosphate to phytoene, whose stimulation directly depends on light stimuli [37]. For instance, UVR8 as the main UV-B receptors, and cryptochromes, as receptors of blue light, are in charge to activate several transcription factors (COP1 protein, constitutive photomorphogenesis 1), which link with HY5 (elongated hypocoty15) as the main PSY stimulator [37]. Furthermore, red and far-red wavelengths are absorbed by phytochromes, which also regulate the photomorphogenesis of carotenoids through the control of phytochrome interacting factors (PIF) [37,38].

Regarding to this behaviour, Pola et al. [39] have recently shown that the exposure to continuous red LEDs is more effective at inducing the accumulation of carotenoids than blue LEDs in mature green chilli peppers exposed to $946 \mathrm{~kJ} \mathrm{~m}^{-2}$ for $3 \mathrm{~d}$ at $30{ }^{\circ} \mathrm{C}$ 
and $75 \% \mathrm{RH}$. This positive effect was also shown in fresh-cut red peppers for $14 \mathrm{~d}$ at $7{ }^{\circ} \mathrm{C}$ and $85 \% \mathrm{RH}$ under $8 \mathrm{~h}$ of $946 \mathrm{~kJ} \mathrm{~m}^{-2}$ red LED in comparison with $630.7 \mathrm{~kJ} \mathrm{~m}^{-2}$ blue LED [33]. Moreover, the exposition to UV-B and UV-C radiation are also demonstrated to affect the expression of genes directly related to carotenogenesis. For instance, UV-B has shown a regulator effect of the initial steps of carotenoid synthesis, stimulating the PSY and lycopene biosynthesis in filamentous cyanobacterium Chlorogloeopsis fritschii PCC 6912 after $9.5 \mathrm{~kJ} \mathrm{~m}^{-2}(4 \mathrm{~h})$ and $37.8 \mathrm{~kJ} \mathrm{~m}^{-2}(4 \mathrm{~h} / \mathrm{d}$ during $4 \mathrm{~d})$ [40]. Similarly, this positive effect of UV-B radiation on carotenoid accumulation has been reported in tobacco leaves after $9.75 \mathrm{~kJ} \mathrm{~m}^{-2}$ for $8 \mathrm{~d}$ [41]. Moreira-Rodríguez et al. [14] also demonstrated an increase in the carotenoid content in broccoli sprouts after $7.16 \mathrm{~kJ} \mathrm{~m}^{-2} \mathrm{UV}-\mathrm{B}$.

Knowing that colour development throughout the postharvest storage is directly related to continuous carotenogenesis during the ripening process [3], the $\Delta \mathrm{E}$ results that were previously mentioned can be linked to the higher carotenoid concentration in FL + BR LED red peppers. Hence, postharvest UV-B treatment of $9 \mathrm{~kJ} \mathrm{~m}^{-2}$ single or combined with a photoperiod of $14 \mathrm{~h} \mathrm{FL}+10 \mathrm{~h}$ BR LED was demonstrated to be a useful tool to increase the carotenoid content in red peppers after the retailing period. Furthermore, it is important to remark that such alternative lighting treatment is able to reduce the operational cost by $\sim 71 \%$ compared to a FL + FL photoperiod according to a fixed price of energy.

\section{Conclusions}

A retail sale period of $4 \mathrm{~d}$ at $20{ }^{\circ} \mathrm{C}$ under $14 \mathrm{~h} \mathrm{FL}+10 \mathrm{~h}$ under blue and red LEDs reported an increase of $\sim 33 \%$ of 13 -cis- $\beta$-carotene, $\sim 24 \%$ of all-trans- $\beta$-carotene, and $\sim 27.5 \%$ of 9 -cis- $\beta$-carotene in red bell peppers when compared to the other conventional photoperiods used in supermarkets (including darkness or FL). Biosynthesis of capsaicinoids, as the major carotenoid compound in red bell peppers, was greatly stimulated after a UV-B postharvest treatment, which was even enhanced after supplementation with the described BR LED photoperiod regarding the conventional retail sale lighting procedures. Therefore, our first proposal is to illuminate peppers with BR LEDs during the retail sale period nights, when supermarkets close, instead of switching off the lights. In this way, an increase in the bioactive content of bell peppers via carotenoid accumulation is induced without compromising quality. Moreover, this proposal can even be included in domestic refrigerators, installing BR LEDs in the fruit and vegetables compartment.

Therefore, our results suggest transferring this technology to the retail sale procedure of bell peppers, and probably other fruit and vegetables, in order to enhance healthiness while decreasing energy and operational costs up to $\sim 70 \%$.

Author Contributions: Conceptualization, F.A.-H. and L.M.-Z.; methodology, N.C. and L.M.-Z.; software, L.M.-Z.; validation, F.A.-H., N.C., and L.M.-Z.; formal analysis, N.C., and L.M.-Z.; investigation, F.A.-H., N.C., and L.M.-Z.; resources, F.A.-H.; data curation, N.C., and L.M.-Z.; writing-original draft preparation, F.A.-H. and L.M.-Z.; writing-review and editing, F.A.-H., N.C., and L.M.-Z.; visualization, F.A.-H.; supervision, F.A.-H.; project administration, F.A.-H.; funding acquisition, F.A.-H. All authors have read and agreed to the published version of the manuscript.

Funding: This research was not funded.

Institutional Review Board Statement: Not applicable.

Informed Consent Statement: Not applicable.

Data Availability Statement: Not applicable.

Acknowledgments: Lorena Martínez-Zamora contract has been co-financed by the European Social Fund (ESF) and the Youth European Initiative (YEI) under the Spanish Seneca Foundation (21322/PDGI/19). Noelia Castillejo was funded by a predoctoral grant (FPU16/04763) from the Spanish Ministry of Education. Authors thanks Tárraga y Henarejos, S.L. for kindly supplying bell peppers and Antoliano Riquelme, Pedro J. Artés and Francisco Artés for the technical assistance during fruit cultivation.

Conflicts of Interest: The authors declare no conflict of interest. 


\section{References}

1. Boeing, H.; Bechthold, A.; Bub, A.; Ellinger, S.; Haller, D.; Kroke, A.; Leschik-Bonnet, E.; Müller, M.J.; Oberritter, H.; Schulze, M.; et al. Critical review: Vegetables and fruit in the prevention of chronic diseases. Eur. J. Nutr. 2012, 51, 637-663. [CrossRef]

2. Wang, X.; Ouyang, Y.; Liu, J.; Zhu, M.; Zhao, G.; Bao, W.; Hu, F.B. Fruit and vegetable consumption and mortality from all causes, cardiovascular disease, and cancer: Systematic review and dose-response meta-analysis of prospective cohort studies. BMJ 2014, 349. [CrossRef]

3. Hassan, N.M.; Yusof, N.A.; Yahaya, A.F.; Rozali, N.N.M.; Othman, R. Carotenoids of Capsicum Fruits: Pigment Profile and Health-Promoting Functional Attributes. Antioxidants 2019, 8, 469. [CrossRef]

4. Miranda-Molina, F.D.; Valle-Guadarrama, S.; Guerra-Ramírez, D.; Arévalo-Galarza, M.D.L.; Pérez-Grajales, M.; Artés-Hernández, F. Quality attributes and antioxidant properties of Serrano chili peppers (Capsicum annuum L.) affected by thermal conditions postharvest. Int. Food Res. J. 2019, 26, 1889-1898.

5. Hamed, M.; Kalita, D.; Bartolo, M.E.; Jayanty, S.S. Capsaicinoids, Polyphenols and Antioxidant Activities of Capsicum annuum: Comparative Study of the Effect of Ripening Stage and Cooking Methods. Antioxidants 2019, 8, 364. [CrossRef] [PubMed]

6. Penella, C.; Calatayud, A. Pepper Crop under Climate Change: Grafting as an Environmental Friendly Strategy. Clim. Resilient Agric. Strateg. Perspect. 2018. [CrossRef]

7. MAPA. Superficies y Producciones de Cultivos 2019. Available online: https://www.mapa.gob.es/es/estadistica/temas/ estadisticas-agrarias/agricultura/superficies-producciones-anuales-cultivos/ (accessed on 2 March 2021).

8. Zhang, X.; Bian, Z.; Yuan, X.; Chen, X.; Lu, C. A review on the effects of light-emitting diode (LED) light on the nutrients of sprouts and microgreens. Trends Food Sci. Technol. 2020, 99, 203-216. [CrossRef]

9. Cisneros-Zevallos, L.; Jacobo-Velázquez, D.A. Controlled Abiotic Stresses Revisited: From Homeostasis through Hormesis to Extreme Stresses and the Impact on Nutraceuticals and Quality during Pre- and Postharvest Applications in Horticultural Crops. J. Agric. Food Chem. 2020, 68, 1877-1879. [CrossRef] [PubMed]

10. Formica-Oliveira, A.C.; Martínez-Hernández, G.B.; Díaz-López, V.; Artés, F.; Artés-Hernández, F. Effects of UV-B and UV-C combination on phenolic compounds biosynthesis in fresh-cut carrots. Postharvest Biol. Technol. 2017, 127, 99-104. [CrossRef]

11. Guidi, L.; Brunetti, C.; Fini, A.; Agati, G.; Ferrini, F.; Gori, A.; Tattini, M. UV radiation promotes flavonoid biosynthesis, while negatively affecting the biosynthesis and the de-epoxidation of xanthophylls: Consequence for photoprotection? Environ. Exp. Bot. 2016, 127, 14-25. [CrossRef]

12. Jenkins, G.I. Signal Transduction in Responses to UV-B Radiation. Annu. Rev. Plant Biol. 2009, 60, 407-431. [CrossRef] [PubMed]

13. León-Chan, R.G.; López-Meyer, M.; Osuna-Enciso, T.; Sañudo-Barajas, J.A.; Heredia, J.B.; León-Félix, J. Low temperature and ultraviolet-B radiation affect chlorophyll content and induce the accumulation of UV-B-absorbing and antioxidant compounds in bell pepper (Capsicum annuum) plants. Environ. Exp. Bot. 2017, 139. [CrossRef]

14. Moreira-Rodríguez, M.; Nair, V.; Benavides, J.; Cisneros-Zevallos, L.; Jacobo-Velázquez, D.A. UVA, UVB Light, and Methyl Jasmonate, Alone or Combined, Redirect the Biosynthesis of Glucosinolates, Phenolics, Carotenoids, and Chlorophylls in Broccoli Sprouts. Int. J. Mol. Sci. 2017, 18, 2330. [CrossRef] [PubMed]

15. Dou, H.; Niu, G.; Gu, M. Pre-Harvest UV-B Radiation and Photosynthetic Photon Flux Density Interactively Affect Plant Photosynthesis, Growth, and Secondary Metabolites Accumulation in Basil (Ocimum Basilicum) Plants. Agronomy 2019,9 , 434. [CrossRef]

16. Schreiner, M.; Martínez-Abaigar, J.; Glaab, J.; Jansen, M. UV-B Induced Secondary Plant Metabolites. Opt. Photon. 2014, 9, 34-37. [CrossRef]

17. Woltering, E.J.; Witkowska, I.M. Effects of pre- and postharvest lighting on quality and shelf life of fresh-cut lettuce. Acta Hortic. 2016. [CrossRef]

18. Jin, P.; Yao, D.; Xu, F.; Wang, H.; Zheng, Y. Effect of light on quality and bioactive compounds in postharvest broccoli florets. Food Chem. 2015, 172, 705-709. [CrossRef]

19. Pintos, F.M.; Hasperué, J.H.; Vicente, A.R.; Rodoni, L.M. Role of white light intensity and photoperiod during retail in broccoli shelf-life. Postharvest Biol. Technol. 2020, 163, 111121. [CrossRef]

20. Loi, M.; Liuzzi, V.C.; Fanelli, F.; De Leonardis, S.; Maria Creanza, T.; Ancona, N.; Paciolla, C.; Mulè, G. Effect of different light-emitting diode (LED) irradiation on the shelf life and phytonutrient content of broccoli (Brassica oleracea L. var. italica). Food Chem. 2019, 283, 206-214. [CrossRef]

21. Hasperué, J.H.; Rodoni, L.M.; Guardianelli, L.M.; Chaves, A.R.; Martínez, G.A. Use of LED light for Brussels sprouts postharvest conservation. Sci. Hortic. 2016, 213, 281-286. [CrossRef]

22. Castillejo, N.; Martínez-Zamora, L.; Gómez, P.A.; Pennisi, G.; Crepaldi, A.; Fernández, J.A.; Orsini, F.; Artés-Hernández, F. Postharvest LED lighting: Effect of red, blue and far red on quality of minimally processed broccoli sprouts. J. Sci. Food Agric. 2021, 101, 44-53. [CrossRef] [PubMed]

23. Pérez-Ambrocio, A.; Guerrero-Beltrán, J.A.; Aparicio-Fernández, X.; Ávila-Sosa, R.; Hernández-Carranza, P.; Cid-Pérez, S.; Ochoa-Velasco, C.E. Effect of blue and ultraviolet-C light irradiation on bioactive compounds and antioxidant capacity of habanero pepper (Capsicum chinense) during refrigeration storage. Postharvest Biol. Technol. 2018, 135, 19-26. [CrossRef] 
24. Formica-Oliveira, A.C.; Martínez-Hernández, G.B.; Díaz-López, V.; Artés, F.; Artés-Hernández, F. Use of postharvest UV-B and $\mathrm{UV}-\mathrm{C}$ radiation treatments to revalorize broccoli byproducts and edible florets. Innov. Food Sci. Emerg. Technol. 2017, 43, 77-83. [CrossRef]

25. Martínez-Zamora, L.; Castillejo, N.; Gómez, P.A.; Artés, F. Amelioration Effect of LED Lighting in the Bioactive Compounds Synthesis during Carrot Sprouting. Agronomy 2021, 11, 304. [CrossRef]

26. Pennisi, G.; Blasioli, S.; Cellini, A.; Maia, L.; Crepaldi, A.; Braschi, I.; Spinelli, F.; Nicola, S.; Fernandez, J.A.; Stanghellini, C.; et al. Unraveling the Role of Red:Blue LED Lights on Resource Use Efficiency and Nutritional Properties of Indoor Grown Sweet Basil. Front. Plant Sci. 2019, 10, 1-15. [CrossRef]

27. Torres-Sánchez, R.; Martínez-Zafra, M.T.; Castillejo, N.; Guillamón-Frutos, A.; Artés-Hernández, F. Real-Time Monitoring System for Shelf Life Estimation of Fruit and Vegetables. Sensors 2020, 20, 1860. [CrossRef] [PubMed]

28. International Organization for Standardization (ISO) 8586. Sensory Analysis-General Guidelines for the Selection, Training and Monitoring of Selected Assessors and Expert Sensory Assessors. Available online: https://www.iso.org/standard/45352.html (accessed on 5 February 2021).

29. Castillejo, N.; Martínez-Hernández, G.B.; Monaco, K.; Gómez, P.A.; Aguayo, E.; Artés, F.; Artés-Hernández, F. Preservation of bioactive compounds of a green vegetable smoothie using short time-high temperature mild thermal treatment. Food Sci. Technol. Int. 2017, 23, 46-60. [CrossRef]

30. Pennisi, G.; Orsini, F.; Castillejo, N.; Gómez, P.A.; Crepaldi, A.; Fernández, J.A.; Egea-Gilabert, C.; Artés-Hernández, F.; Gianquinto, G. Spectral composition from led lighting during storage affects nutraceuticals and safety attributes of fresh-cut red chard (Beta vulgaris) and rocket (Diplotaxis tenuifolia) leaves. Postharvest Biol. Technol. 2021, 175, 111500. [CrossRef]

31. Kasim, M.U.; Kasim, R. The effects of ultraviolet B (UV-B) irradiation on color quality and decay rate of Capia pepper during postharvest storage. Food Sci. Technol. 2018. [CrossRef]

32. Villaseñor-Aguilar, M.J.; Bravo-Sánchez, M.G.; Padilla-Medina, J.A.; Vázquez-Vera, J.L.; Guevara-González, R.G.; GarcíaRodríguez, F.J.; Barranco-Gutiérrez, A.I. A Maturity Estimation of Bell Pepper (Capsicum annuum L.) by Artificial Vision System for Quality Control. Appl. Sci. 2020, 10, 5097. [CrossRef]

33. Maroga, G.M.; Soundy, P.; Sivakumar, D. Different Postharvest Responses of Fresh-Cut Sweet Peppers Related to Quality and Antioxidant and Phenylalanine Ammonia Lyase Activities during Exposure to Light-Emitting Diode Treatments. Foods 2019, 8, 359. [CrossRef] [PubMed]

34. Vicente, A.R.; Pineda, C.; Lemoine, L.; Civello, P.M.; Martinez, G.A.; Chaves, A.R. UV-C treatments reduce decay, retain quality and alleviate chilling injury in pepper. Postharvest Biol. Technol. 2005. [CrossRef]

35. Cuvi, M.J.A.; Vicente, A.R.; Concellón, A.; Chaves, A.R. Changes in red pepper antioxidants as affected by UV-C treatments and storage at chilling temperatures. LWT Food Sci. Technol. 2011, 44, 1666-1671. [CrossRef]

36. Del Gómez-García, M.R.; Ochoa-Alejo, N. Biochemistry and Molecular Biology of Carotenoid Biosynthesis in Chili Peppers (Capsicum spp.). Int. J. Mol. Sci. 2013, 14, 19025-19053. [CrossRef]

37. Tian, S.L.; Li, L.; Shah, S.N.M.; Gong, Z.H. The relationship between red fruit colour formation and key genes of capsanthin biosynthesis pathway in Capsicum annuum. Biol. Plant. 2015. [CrossRef]

38. Llorente, B.; Martinez-Garcia, J.F.; Stange, C.; Rodriguez-Concepcion, M. Illuminating colors: Regulation of carotenoid biosynthesis and accumulation by light. Curr. Opin. Plant Biol. 2017, 37, 49-55. [CrossRef]

39. Pola, W.; Sugaya, S.; Photchanachai, S. Color Development and Phytochemical Changes in Mature Green Chili (Capsicum annuum L.) Exposed to Red and Blue Light-Emitting Diodes. J. Agric. Food Chem. 2020. [CrossRef]

40. Llewellyn, C.A.; Airs, R.L.; Farnham, G.; Greig, C. Synthesis, Regulation and Degradation of Carotenoids under Low Level UV-B Radiation in the Filamentous Cyanobacterium Chlorogloeopsis fritschii PCC 6912. Front. Microbiol. 2020, 11, 163. [CrossRef]

41. Shen, J.; Jiang, C.Q.; Yan, Y.F.; Liu, B.R.; Zu, C.L. Effect of increased UV-B radiation on carotenoid accumulation and total antioxidant capacity in tobacco (Nicotiana tabacum L.) leaves. Genet. Mol. Res. 2017, 16. [CrossRef] [PubMed] 